\title{
Hydrocephalus and CSF Disorders
}

\author{
Richard Farb and Àlex Rovira
}

\section{Learning Objectives}

- To know CSF physiology and the different types of hydrocephalus

- To recognize the radiological features of the different types of hydrocephalus

- To be able to recognize the imaging features that distinguish normal pressure hydrocephalus from other causes of ventriculomegaly in the adult population

- To recognize imaging findings in disorders of intracranial pressure and how they impact direct subsequent management

\subsection{Introduction}

Modern imaging techniques play an essential role for understanding of the anatomy of the cerebrospinal fluid (CSF) spaces and ventricular system, as well as the hydrodynamics of CSF flow. These principles are important to the understanding of pathological processes affecting the ventricles and CSF spaces, and abnormalities induced by changes in the intracranial pressure.

The classical model of CSF hydrodynamics presumes that CSF is produced primarily in the choroid plexus epithelium (and to a lesser degree in the ependymal cells) at a rate of approximately $0.2-0.6 \mathrm{~mL} / \mathrm{min}$ and $400-600 /$ day. However, numerous evidences indicate that CSF is also produced throughout the entire CSF-interstitial fluid functional unit across the walls of central nervous system (CNS) blood capillaries [1].

\section{R. Farb}

Division of Neuroradiology, Toronto Western Hospital, University

Health Network, University of Toronto, Toronto, ON, Canada

e-mail: richard.farb@uhn.ca

À. Rovira $(\bowtie)$

Section of Neuroradiology, Hospital Universitari Vall d'Hebron,

Barcelona, Spain

e-mail: alex.rovira@idi.gencat.cat
Since the total volume of CSF in the ventricles and subarachnoidal spaces (SAS) averages approximately $150 \mathrm{~mL}$, a threefold turnover of CSF occurs daily, explaining the presence of a bulk flow of CSF from the sites of origin to the sites of absorption. This bulk flow of CSF starts in the lateral ventricles, passes through the third and fourth ventricles and, via the foramina of Luschka and the foramen of Magendie into the cerebellopontine angle and prepontine cisterns and the vallecula of the cisterna magna. From the basilar cisterns, the bulk flow of CSF continues cephalad along two major routes: a ventral route through the interpeduncular and pre-chiasmatic cisterns and a dorsomedial route through the ambient cisterns and the cisterna magna. The bulk flow also extends downward into the spinal canal dorsal to the cord to the lumbar thecal sac and upward in front of the cord to the basilar cistern.

CSF is absorbed not only via the arachnoid villi that are ideally situated to drain CSF from the SAS into the major dural sinuses, but also through the lymphatic system, and through the glymphatic system in the brain parenchyma, (extracellular fluid spaces that are in communication with the brain capillaries). In fact, it is widely accepted that the vast majority of CSF is absorbed through the capillary network of the CNS [1,2].

There is an increasing body of evidence indicating that superimposed on the low-velocity bulk flow of CSF, which is almost insignificant $(4 \mathrm{~mL} / \mathrm{min})$, there is a high-velocity oscillatory CSF motion $(700 \mathrm{~mL} / \mathrm{min})$, whose main driving force is the cardiac-related intracranial arterial expansion. MRI is well suited for demonstrating this oscillatory high-velocity craniospinal CSF flow in a noninvasive manner. Cardiac-gated flow-sensitive phase-contrast cine MRI (PC-MRI) is probably the method of choice, since it allows qualitative and quantitative analysis of oscillatory CSF movement in normal and abnormal situations. This MRI technique uses a two-dimensional (2D) cine phase-contrast sequence that sensitizes the images to velocity changes in a specific direction while canceling signals from stationary protons and from motion in other directions [3] (Fig. 2.1). 

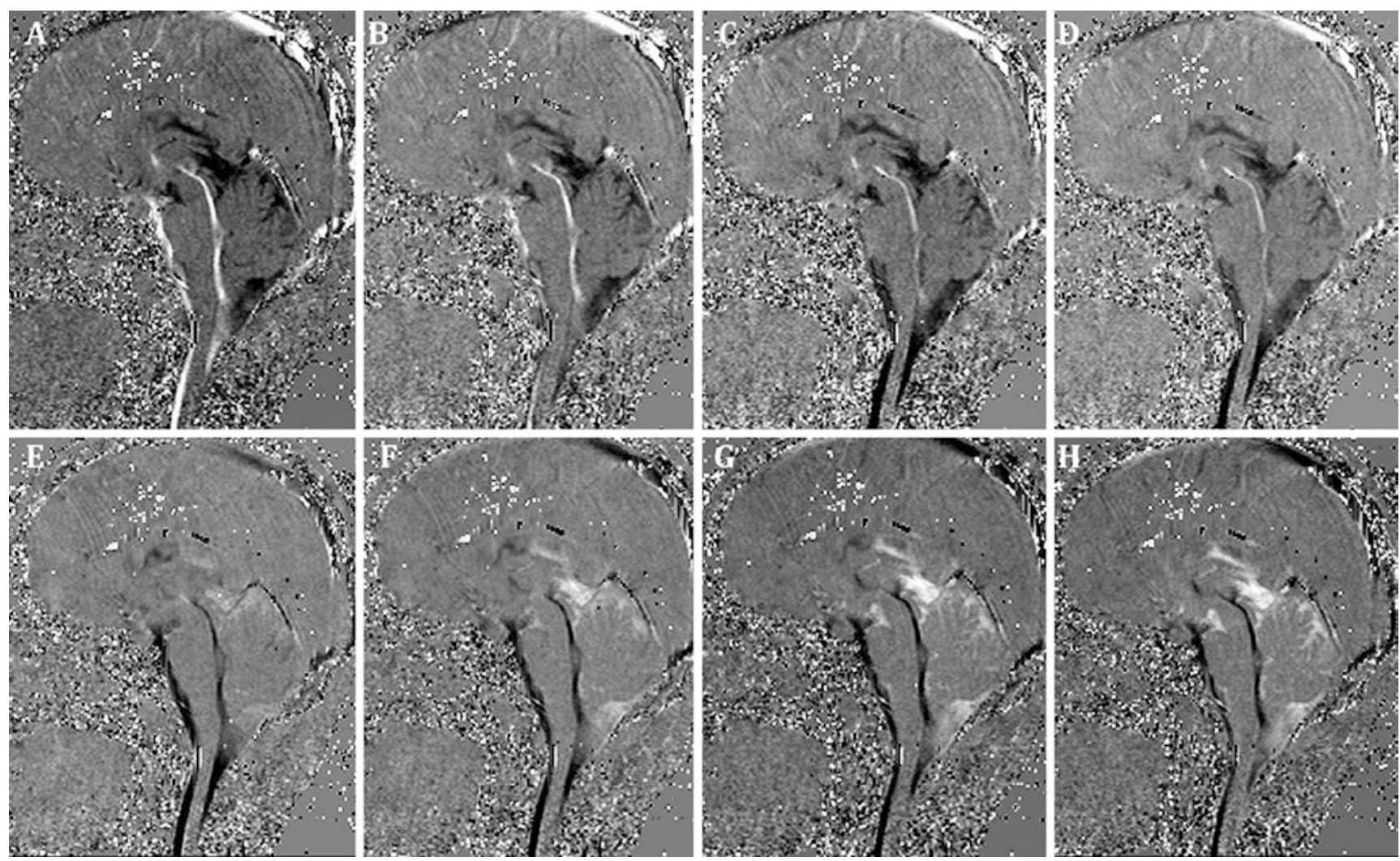

Fig. 2.1 Cine phase-contrast MRI in a normal subject. (a-h) Normal intracranial and upper cervical spine cerebrospinal fluid oscillatory flow pattern: Midsagittal phase-contrast flow-sensitive images showing the whole cardiac cycle. The flow is sensitized craniocaudally. Caudocranial flow is represented in black, and craniocaudal in white. Antegrade flow is initiated from the lower fourth ventricle and cisterna magna. Caudal flow begins in the

posterior third ventricle, aqueduct of Sylvius, and upper fourth ventricle. As pulsation through the aqueduct continues caudally, pulsation in the cisterna magna becomes quiescent, and then reverses to pulse cephalically. All flow shows cephalic pulsation. Reproduced from Rovira A. Communicating Hydrocephalus. Normal Pressure Hydrocephalus. In F. Barkhof et al. (eds.), Clinical Neuroradiology, @ Springer Nature Switzerland AG 2019

In this chapter we will review the clinical and imaging features of the different types of hydrocephalus, both in adults and in children, and other idiopathic disorders associated with an abnormal intracranial pressure, such as idiopathic intracranial hypertension and spontaneous intracranial hypotension.

\section{Key Points}

- The production of CSF mostly takes place within the CNS capillaries: filtration of water volume through the capillary walls into the surrounding brain tissue. The choroid plexuses are a less relevant place for this process.

- CSF absorption takes place at different sites, and its capacity far exceeds CSF production.

- A high-velocity oscillatory CSF motion driven by cardiac-related intracranial arterial expansion can be identified and quantified non-invasively with different MRI techniques.

\subsection{Obstructive Hydrocephalus in Adults}

The term obstructive hydrocephalus usually indicates noncommunicating hydrocephalus, and occurs when the flow of CSF is blocked along one or more of the narrow apertures connecting the ventricles [5].

Usually CT is the first-line imaging method, especially in the emergency setting. Although this technique is able to show signs of acute as well as chronic hydrocephalus, MRI is the method of choice for detailed assessment of this condition. Contrastenhanced T1-weighted imaging is not required for the diagnosis unless there is a suspicion of a neoplastic lesion or an inflammatory process. The main purpose of the diagnostic management of the patient with obstructive hydrocephalus is to find an obstacle impeding the CSF flow within the ventricular system [6].

When conventional MRI sequences are not able to clearly find the obstructive lesion, some new MR techniques may help for determining the mechanism producing the hydrocephalus. These new techniques include PC-MRI and 3D heavily T2-weighted steady-state sequences (3DT2WSS) [5]. 

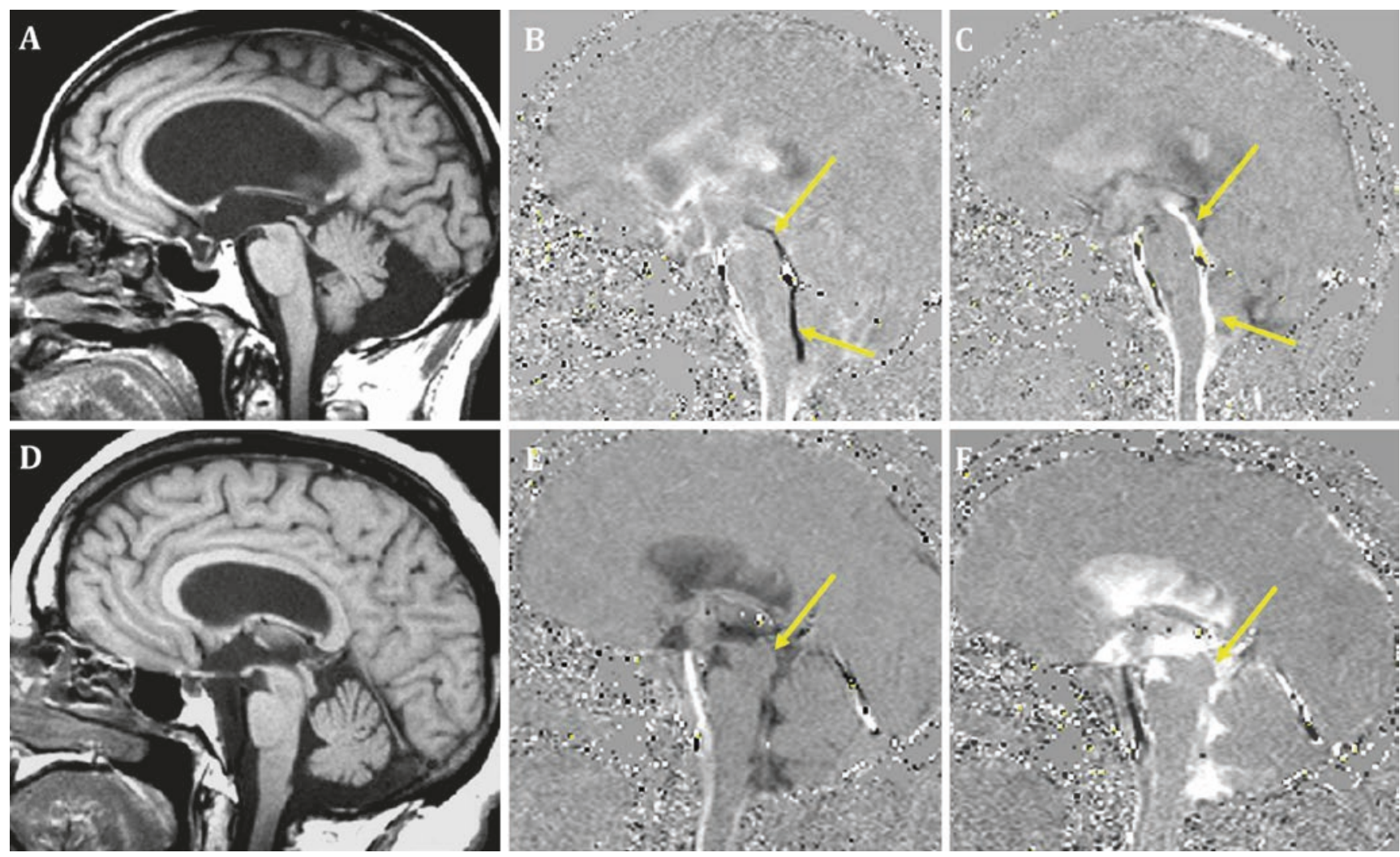

Fig. 2.2 Brain MRI in a patient with communicating hydrocephalus $(\mathbf{a}-\mathbf{c})$ and in a patient with a non-communicating hydrocephalus secondary to aqueductal stenosis (d-f). Midsagittal T1-weighted and two representative frames of the diastolic and systolic phases of a phasecontrast flow-sensitive MRI sequence $(\mathbf{b}, \mathbf{c}, \mathbf{e}, \mathbf{f})$. Observe the presence of a normal craniocaudal cerebrospinal fluid flow through the aqueduct

PC-MRI enables quantitative and qualitative assessment of the CSF circulation (Fig. 2.2), while 3DT2WSS sequences such as DRIVE (Philips), CISS (Siemens), and FIESTA (GE) are useful for accurate anatomic evaluation and are highly recommended in patients with suspicion of aqueductal pathology or intraventricular adhesions. Alternatively, to PC-MRI, 3D T2-weighted turbo spin-echo sequences with variable flip angle (3DT2WTSE) can be used (Siemens: SPACE, GE: CUBE, Philips: VISTA) for detecting the high-velocity CSF bulk flow, as are strongly susceptible to CSF flow effects. This technique is noninvasive, less sensitive to artifacts, and enables scanning of the whole skull using isotropic voxels with an acceptable acquisition time [5] (Figs. 2.3 and 2.4).

\subsubsection{Radiological Features of Hydrocephalus}

The typical radiological features of any kind of hydrocephalus are:

- Enlargement of the ventricles (Evans index $>0.3$ ) out of proportion of the size of SAS (cortical sulci appear dis- in the patient with a communicating hydrocephalus, and the absence of the CSF flow in the patient with aqueductal stenosis. Reproduced from Rovira A. Communicating Hydrocephalus. Normal Pressure Hydrocephalus. In F. Barkhof et al. (eds.), Clinical Neuroradiology, (C) Springer Nature Switzerland AG 2019

proportionately narrowed), with ballooning of the frontal and posterior ventricular horns

- Dilatation of temporal ventricular horns

- Dilatation of the third ventricular recesses

- Reduced mamillopontine distance

- Upward bowing and thinning of the corpus callosum

- Hyperintense on MRI (hypodense on CT) areas in the periventricular white matter, suggestive features of acute hydrocephalus.

Typical obstacle sites in obstructive hydrocephalus are the foramen of Monro, the cerebral aqueduct, the fourth ventricular outlets (Luschka and Magendie foramina), and the foramen magnum.

\subsubsection{Foramen of Monro Obstruction}

Several lesions growing in the region adjacent to Monro foramina might produce obstructive hydrocephalus. These include intra- and periventricular cysts such as arachnoid, colloid, dermoid, epidermoid cysts, tumors (ependymomas, 

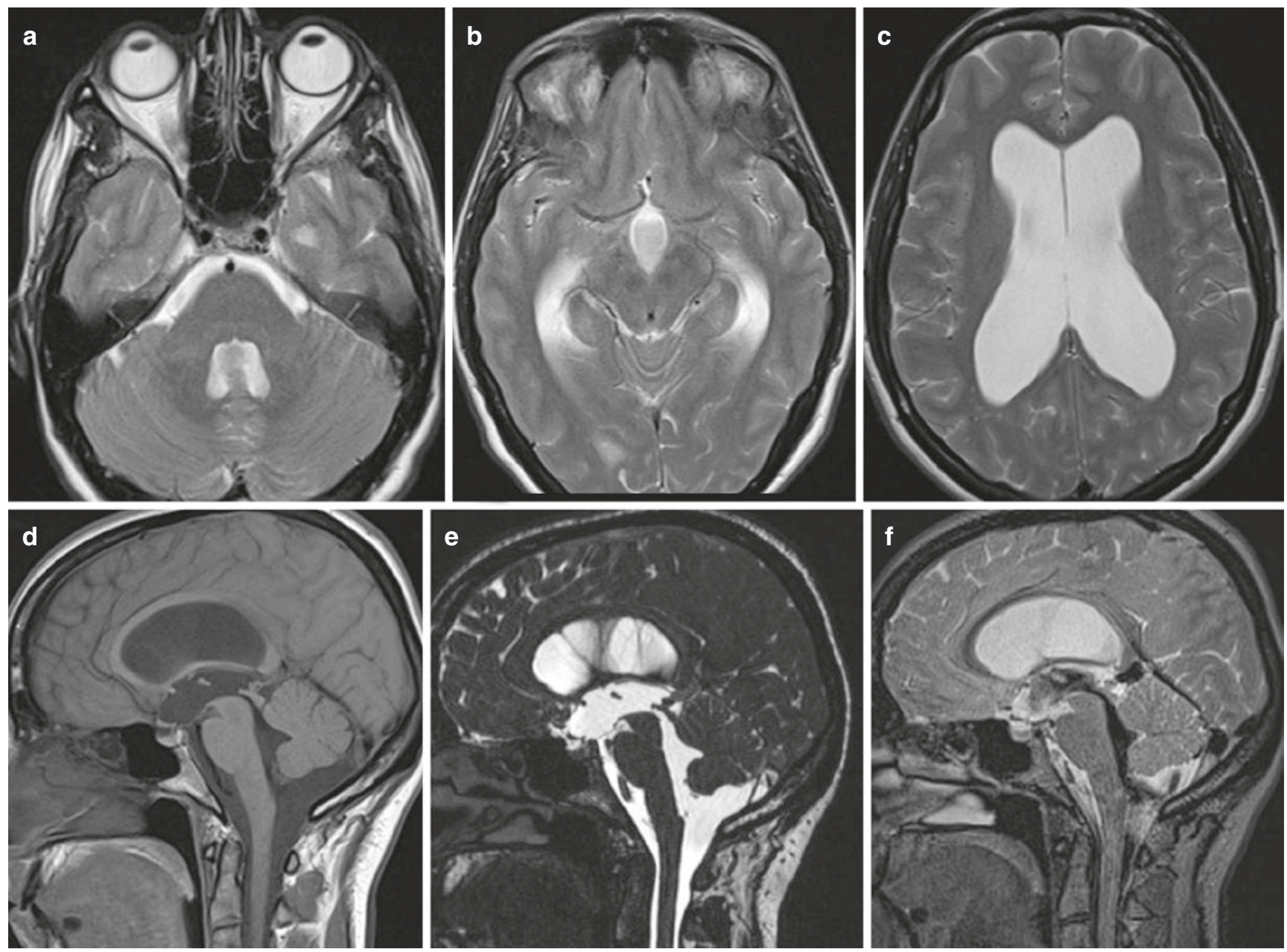

Fig. 2.3 Brain MRI in a patient with communicating hydrocephalus. Axial T2-weighted images (a-c) show ventriculomegaly involving the supratentorial and infratentorial ventricular cavities. Observe the presence of flow voids inside the aqueduct and fourth ventricle. Midsagittal T1-weighted (d) 3DT2WSS sequence (CISS) (e) and 3DT2WTSE
(SPACE) (f) images show a morphological normal aqueduct with marked flow void, better seen on the SPACE sequence. Reproduced from Rovira A. Communicating Hydrocephalus. Normal Pressure Hydrocephalus. In F. Barkhof et al. (eds.), Clinical Neuroradiology, (C) Springer Nature Switzerland AG 2019 subependymomas, central neurocytomas, or glial tumors), as well as adhesions. Not infrequently these lesions may lead to unilateral ventriculomegaly. Also, unilateral mass lesions producing midline shift might eventually obstruct the contralateral foramen of Monro causing contralateral ventricular dilatation as well as compression of the ipsilateral lateral ventricle.

The most common pathology located in this area and responsible for hydrocephalus is a colloid cyst, which are slow-growing, benign cystic lesions, typically located in the anterior part of the third ventricle. These usually oval or round lesions are mostly hyperdense on unenhanced $\mathrm{CT}$ in comparison to the gray matter, although in one-third of cases are hypo- or isodense. On MRI, the signal intensity is quite variable, depending on their protein content, but approxi- mately $50 \%$ of them are hyperintense on T1-weighted sequences and hypointense on T2-weighted sequences.

Subependymal giant cell astrocytomas (SEGAs) are the most common brain tumors in tuberosus sclerosis complex occurring in about one out of every ten patients. These tumors are low-grade (World Health Organization [WHO] grade 1), mixed, glioneuronal, and highly vascularized neoplasms predominantly located near the foramen of Monro, and frequently with bilateral distribution. They represent a significant cause of morbidity and mortality because of the risk of sudden death from acute hydrocephalus, the risk of which is directly proportional to their volume. On CT, SEGAs appear as iso/hyperdense masses showing frequent calcification. On MRI, they have hypo/isointense signal on T1-weighted sequences and 
Fig. 2.4 Brain MRI in a patient with communicating hydrocephalus $(\mathbf{a}, \mathbf{b})$ and in a patient with a noncommunicating hydrocephalus secondary to aqueductal stenosis (c, d). Midsagittal 3DT2WSS sequence (CISS) (a, c) and 3DT2WTSE sequence (SPACE) (b, d) images. Observe the presence of a normal morphology of the aqueduct and normal cerebrospinal fluid flow through the aqueduct in the patient with a communicating hydrocephalus (arrows), and the thickened mesencephalic tectum (probable tectum glioma) and absence of the CSF flow in the patient with aqueductal stenosis (arrows). Note the presence of normal CSF flow through the Magendie foramen (short arrow). Reproduced from Rovira A. Communicating Hydrocephalus. Normal Pressure Hydrocephalus. In F. Barkhof et al. (eds.), Clinical Neuroradiology, (C) Springer Nature Switzerland AG 2019
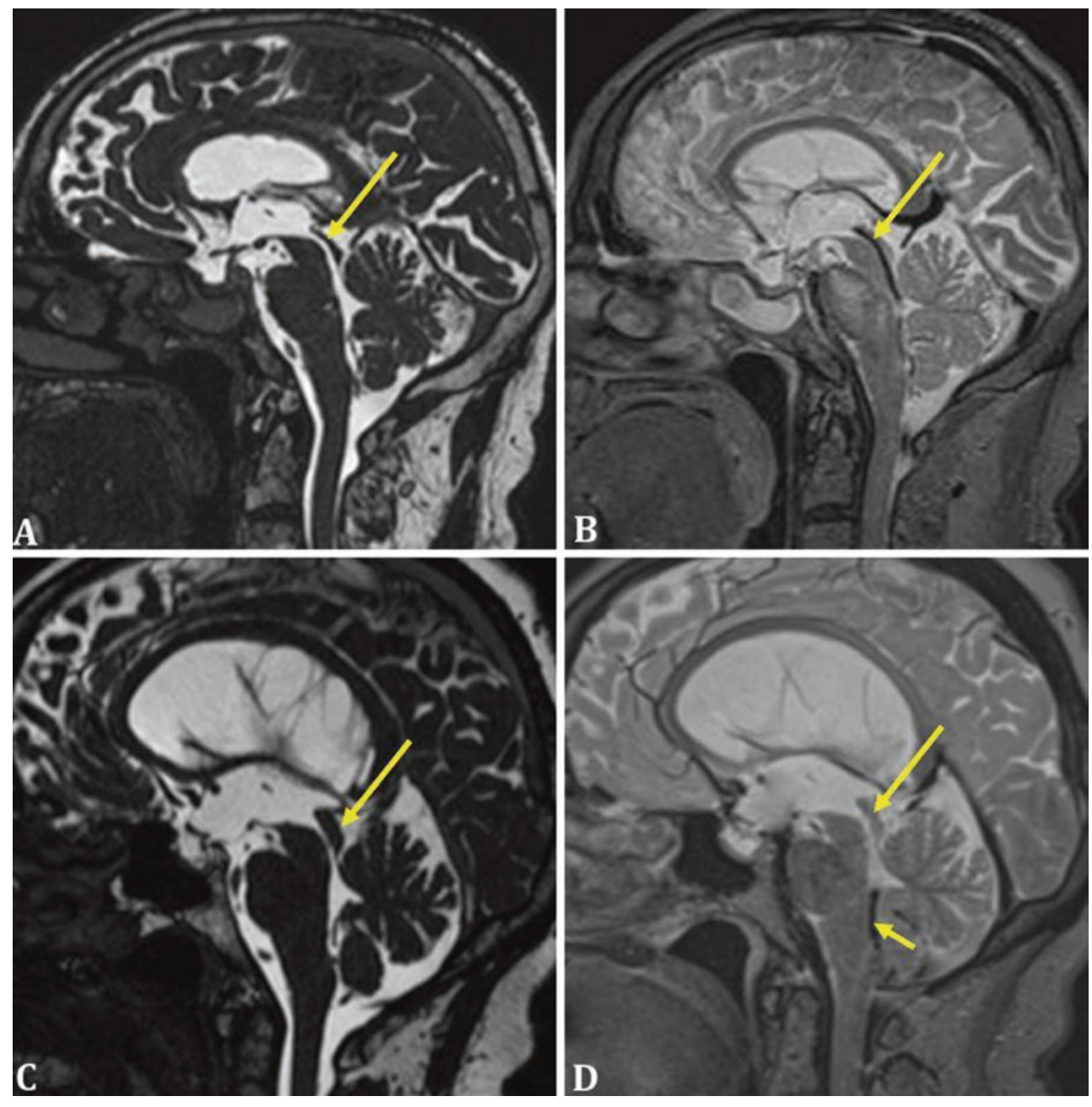

hyperintense signal on T2-weighted sequences, presenting intense, but inhomogeneous, contrast enhancement.

\subsubsection{Cerebral Aqueduct}

Aqueductal stenosis, one of the most common causes of obstructive hydrocephalus both in children and adults, can be divided into congenital and acquired. The most frequent acquired lesion resulting in aqueductal stenosis includes pineal region tumors (pineal gland tumors, tectum-tegmentum gliomas, tentorial meningiomas, metastasis), cerebral vascular malformations (e.g., vein of Galen aneurysm), adjacent intracranial hemorrhage, and infections (meningitis/ventriculitis) (Fig. 2.4). The congenital causes may be aqueductal webs or diaphragms. The aqueductal web is a membrane formation whose origin may be either congenital or inflammatory. It has been hypothesized that it might be caused by a small glial occlusion of the caudal aqueduct with the subsequent development of a sheet of tissue due to extended pressure and dilatation of the canal above. On MRI sequences, enlargement of the lateral and third ventricles, without dilatation of fourth ventricle is a key imaging feature. The aqueduct may show funneling superiorly.

\subsubsection{Trapped Fourth Ventricle}

Obstruction of all CSF pathways of the fourth ventricle, including foramina of Luschka and Magendie, as well as aqueduct, produces and isolated enlargement of this cavity known as the trapped fourth ventricle. This is a rare clinical condition most often associated with previous ventricular shunting for hydrocephalus [7], infection, intraventricular hemorrhage, and postinflammatory changes after posterior fossa surgery (Fig. 2.5). The clinical symptoms include nausea, vomiting, and cranial nerves palsy as a result of cerebellar and brain stem compression. Treatment options include placement of a fourth ventriculoperi- 
Fig. 2.5 Sagittal T1- (a) and T2-weighted (b), and coronal T2-FLAIR (c) brain MRI in an 8-year-old girl with congenital hydrocephalus secondary to prenatal infection treated with ventricular shunting. Observe the presence of web-like membranes (arrows in b) at the level of the aqueduct and Magendie foramen, producing a trapped fourth ventricle. (Courtesy of Antoni Rovira. Parc Tauli. Sabadell. Spain)
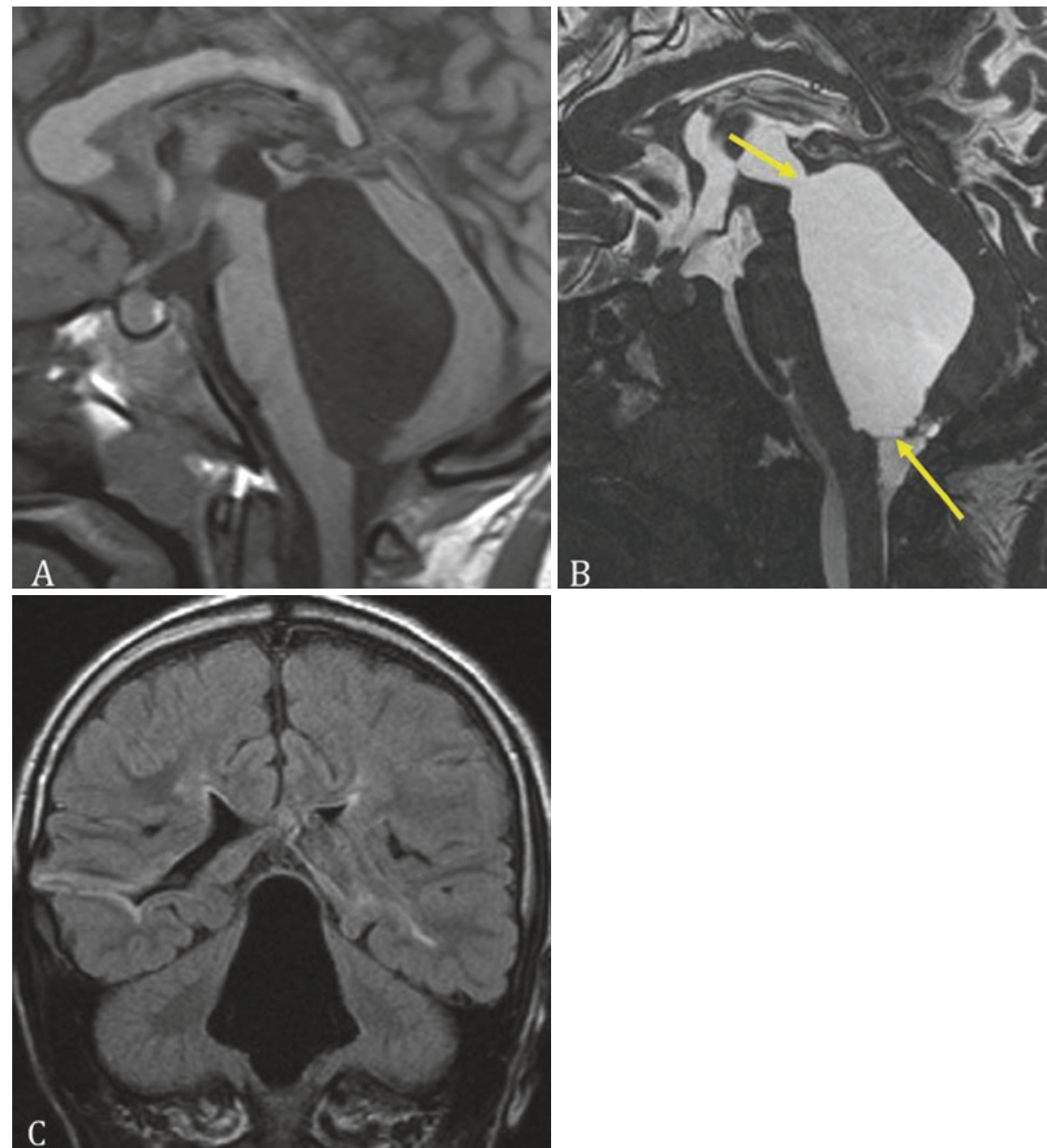

toneal shunt, endoscopic aqueductoplasty, as well as interventriculostomy or open fenestration via suboccipital craniotomy.

\subsubsection{Fourth Ventricular Outlets and Foramen Magnum}

Obstruction of the fourth ventricle outlets (foramina of Luschka and Magendie) and foramen magnum are caused by hemorrhage, meningitis, and extrinsically compressing lesions including tumors (cerebellar metastases, meningiomas, hemangioblastomas, astrocytomas, medulloblastomas) and craniocervical developmental malformation involving the cranio-cervical junction (osteochondrodysplasia, metabolic diseases, developmental abnormalities, and Chiari malformations) [5]. Acute/subacute cerebellar infarcts are also a common cause in the adult population [5]. Primary obstruction of the foramina of Magendie and Luschka by the presence of web-like membranes is an uncommon cause of non-communicating hydrocephalus. 3DT2WSS sequences are particularly useful in the identification of these thin webs.

\section{Key Points}

- 3DT2WSS sequences are useful for accurate anatomic evaluation of the ventricular system, and are highly recommended in patients with suspicion of non-communicating hydrocephalus.

- The inclusion in the imaging protocol of CSF-flow sensitive sequences, such as PC-MRI and3D-T2WTSE, is highly recommended for detecting the normal highvelocity CSF flow through narrower segments of the ventricular system.

- Contrast-enhanced T1-weighted imaging should be obtained when there is a suspicion of a neoplastic lesion or an inflammatory process as the causative mechanism of non-communicating hydrocephalus. 


\subsection{Hydrocephalus in Children}

The etiology and prevalence of pediatric hydrocephalus varies widely throughout the world. In the developing world, neonatal infections account for most cases of hydrocephalus, while in developed regions the more commonly seen etiologies of hydrocephalus include parenchymal hemorrhage, congenital aqueduct stenosis, myelomeningocele, and posterior fossa tumors. The prevalence of hydrocephalus has been estimated to be at least 1 in 1000 births [8, 9].

Etiologies for hydrocephalus in the pediatric populations can also be generally categorized as either congenital or acquired entities. Congenital etiologies include aqueduct stenosis, neural tube defects, and posterior fossa malformations (e.g., vein of Galen malformation obstructing flow at the aqueduct). Acquired etiologies include: subarachnoid or intraventricular hemorrhage or infection, brain tumors, choroid plexus papilloma resulting in overproduction of CSF, and extrinsic venous obstruction.

In the infant, hydrocephalus may present with macrocephaly, irritability, and bulging of the fontanelles. In the older child hydrocephalus typically presents with signs and symptoms of elevated intracranial pressure including headache, vomiting, and delay in developmental milestones.

Management of hydrocephalus consists of treatment of the underlying primary abnormality such as tumor or hemorrhage and CSF diversion procedures with placement of ventriculoperitoneal shunting devices. Newer methods of endoscopic third ventriculostomy (ETV) and choroid plexus cauterization (CPC) have developed since the 1990s and represent an alternative treatment for chronic hydrocephalus. A third ventriculostomy involves a small craniotomy usually through the right frontal region and placement of an endoscope into the right frontal horn, through the foramen of Monro toward the floor of the third ventricle where a small opening is cut in the floor of the third ventricle allowing direct communication with the prepontine cistern. ETV along with CPC via the same endoscopic procedure has shown promising results in early trials.

Complications of long-term shunting include perioperative morbidity and mortality, shunt infection (5-9\% mostly occurring within 3 months of surgery) and shunt malfunction (occurring in $40 \%$ of children within the first 2 years after shunt placement). Interestingly use of valvular apparatus coupled to the silastic catheter has been shown to be ineffective in randomized control trials to decrease the rate of shunt failure.

MRI is the current modality of choice for assessing pediatric hydrocephalus and the effects that might produce in the developing brain. This includes evaluation for size and contour of the ventricles and SAS, presence or absence of interstitial periventricular edema, and identification of the normal CSF aqueductal flow void. Imaging is obviously also indispensable in evaluation for the complications associated with surgical management of hydrocephalus. And, following ETV, can also demonstrate patency of flow across the surgical defect, mainly using flow-sensitive MRI sequences, such as PC-MRI and 3DT2WTSE [10].

\section{Key Point}

- Neonatal infections account for most cases of hydrocephalus in developing regions, while in developed regions the most common causes include parenchymal hemorrhage, congenital aqueduct stenosis, myelomeningocele, and posterior fossa tumors.

\subsection{Communicating Hydrocephalus}

Communicating hydrocephalus is defined as a CSF flow circulation abnormality outside the ventricular system that produces an increase in the ventricular size. Most cases are secondary to obstruction of CSF flow between the basal cisterns and brain convexity, and include common conditions such as subarachnoid hemorrhage, bacterial and aseptic meningitis, and leptomeningeal carcinomatosis. This type of hydrocephalus could be better classified as communicating with obstruction, to differentiate them from idiopathic normal pressure hydrocephalus (iNPH), in which there is not objective obstruction of CSF circulation and absorption [4].

\subsubsection{Communicating Hydrocephalus with Obstruction}

- Subarachnoid hemorrhage: Acute and chronic hydrocephalus is one of the major complications after subarachnoid hemorrhage, with a reported incidence of $20-30 \%$. The hydrocephalus is produced as a consequence of blockage of the SAS that produces impairment of CSF circulation and absorption. The acute form is more common and usually develops within few days after the hemorrhage.

- Meningitis: Bacterial meningitis may be complicated with communicating hydrocephalus, which is associated with poor neurologic outcome. The causative mechanism includes the blockage of SAS by exudates and high protein levels at basal cisterns or convexity that prevent normal CSF circulation and absorption. Less frequently, exudates can block the CSF flow at fourth ventricle exits (Magendie and Luschka foramina), resulting in a noncommunicating type of hydrocephalus. Aseptic meningitis, which could be infective (viral or fungal) or secondary to systemic conditions such as autoimmune diseases or neurosarcoidosis, might produce a communicating hydrocephalus induced by similar mechanisms, but usually 
develops subacutely over a period of weeks to months, and is associated with a lower morbi-mortality compared to the most common hydrocephalus secondary to bacterial meningitis. CT or MRI is rarely indicated for the diagnosis of meningitis, but should be immediately performed in those patients with clinical suspicious of complications (e.g., signs of increased intracranial pressure).

- Leptomeningeal carcinomatosis: Both primary (medulloblastoma, ependymoma, glioblastoma, and oligodendroglioma) and secondary tumors (lymphoma, lung and breast cancers, melanoma) may spread through the SAS (leptomeningeal carcinomatosis). In some cases, the clinical presentation is secondary to impairment of CSF flow with development of communicating hydrocephalus. Contrast-enhanced T1-weighted and T2-FLAIR sequences should be added in patients with communicating hydrocephalus of unknown origin, particularly when the clinical symptoms (e.g., headache) developed acutely or in patients with a known history of cancer.

- Vestibular schwannomas: Hydrocephalus is relatively common in patients with vestibular schwannomas. Although obstructive hydrocephalus due to mechanical compression of the fourth ventricle or cerebral aqueduct by the tumor is demonstrated in some patients, communicating hydrocephalus is more frequent. This latter type of hydrocephalus could be explained by a compromise of CSF absorption by protein leakage from the tumor.

\section{Key Points}

- The term communicating hydrocephalus, better defined as communicating with obstruction, must be differentiated from iNPH, in which there is not objective obstruction of CSF circulation and absorption.

- Common causes of this form of hydrocephalus in the adult population include subarachnoid hemorrhage, bacterial and aseptic meningitis, and leptomeningeal carcinomatosis.

\subsubsection{Normal Pressure Hydrocephalus}

Normal pressure hydrocephalus (NPH) is a syndrome initially identified in 1957 by Solomon Hakim characterized by the triad of gait disturbance, mental deterioration, and urinary incontinence, which are associated with enlargement of the ventricular system and normal CSF pressure. It is difficult to establish the prevalence of NPH due to the non-specific nature of the symptoms, but it has been estimated to be 181.7 per 100,000 people in the 70-79 years age group. About 50\% of cases with NPH have a known cause (secondary or symptomatic NPH [sNPH]), such as meningitis, subarachnoid hemorrhage, or cranial trauma, while the remaining $50 \%$ of cases are idiopathic (iNPH), usually presenting in the seventh decade of life.

The classical concept that NPH is simply a form of CSF circulation disorder, involving an imbalance between CSF production, and reabsorption, is probably not valid. In fact, unlike other forms of hydrocephalus, the CSF pressure is not abnormally raised, which implies that such a simplistic hydrodynamic theory would be insufficient to explain the pathophysiology of this condition. Other factors might contribute to the development of NPH. Chronic hypertension and white matter disease (which may naturally occur in the aging brain) may lead to periventricular ischemia that increases the compliance of the ventricular wall and causes gradual ventricular enlargement. Alternatively, periventricular ischemia may also lead to locally increased venous resistance that may lead to decreased CSF absorption and ventricular enlargement. Finally, diminished vascular (arterial) compliance that frequently occurs in the aging brain might produce a redistribution of systolic vascular pulsations, which are then transmitted directly into the ventricular system, progressively increasing its size.

iNPH is characterized clinically by gait and balance impairment, cognitive deterioration, and urinary incontinence, and radiologically by a communicating ventricular enlargement (Evans Index $>0.3$ ), in the context of a CSF opening pressure of $<24 \mathrm{~cm}$ of $\mathrm{H}_{2} \mathrm{O}$ (in recumbent position). However, the complete triad appears only in 50-60\% of iNPH patients.

The diagnosis of NPH is a challenging process. Initially the diagnosis is suspected on the basis of the history, neurological examination, and interpretation of CT and/or MRI scans. MRI is particularly useful for establishing that a communicating hydrocephalus is present, but may also be effective in identifying patients who will respond to shunting. Exclusion of a noncommunicating hydrocephalus is the first radiological objective in the diagnosis of iNPH. Combination of 3DT2WSS such as CISS (constructive interference steady state) that offer superb anatomical information, and 3DT2WTSE sequences such as SPACE (sampling perfection with application optimized contrast using different flip angle evolutions), which is highly sensitive to CSF flow, provides an easy evaluation of CSF pathway and circulation to establish the patency of CSF flow [11, 12].

A simple way to establish the diagnosis of ventriculomegaly is by means of the Evans index (EI), an indirect linear measurement of ventricular size. It has been established that in normal adults, the EI is $<0.3$, and therefore values $>0.3$ has been used for an objective form to diagnose ventriculomegaly. $\mathrm{EI}$ is not specific for iNPH, as increases with ventriculomegaly independently of its cause. Other morphological features of the ventricular system such as bilateral enlargement of the temporal horns not attributable to hippocampal atrophy, and a dilated third ventricle, have been described as typical findings in both iNPH and obstructive hydrocephalus, but it is not clear the value of these features both for establishing the diagnosis of iNPH or to predict shunt response. 
The callosal angle measured on MRI is determined as the angle between the medial superior borders of the left and right lateral ventricles, from a coronal image obtained at the level of the posterior commissure, perpendicular to a plane that intersected the anterior and posterior commissure. The CA in patients with iNPH is significantly smaller than those with Alzheimer disease or healthy controls, and a value $<90$ $100^{\circ}$ has been used to discriminate between these two groups of subjects with a high accuracy. This accuracy is improved when combining both the CA and the EI $(>0.30-0.32)$. The $\mathrm{CA}$ has also been used to predict shunt-responders, as these patients have a significantly smaller mean preoperative CA compared with non-responders, using $63^{\circ}$ as the cut-off value to achieve the best prognostic accuracy [13].

Narrow cortical sulci at the high convexity (parietal high convexity and medial surface) is a typical finding in patients with NPH. This finding, better identified on coronal MRI or CT scans, is frequently associated with wide Sylvian fissures. This widening, although not uncommon in Alzheimer's disease, is more pronounced and frequent in iNPH. These structural brain changes have been grouped under the term "disproportionately enlarged subarachnoid space hydrocephalus" (DESH), which refers to the combination of ventriculomegaly with narrow high convexity and medial subarachnoid spaces and enlarged Sylvian fissures (disproportionate distribution of the CSF between the inferior and superior subarachnoid spaces), and is present in a high proportion of patients with a final diagnosis of iNPH (Fig. 2.6).

The DESH pattern is extensively used for iNPH diagnosis and predicting shunt response, without the need of a CSF tap test or other invasive procedures [14].
Fig. 2.6 Idiopathic normal pressure hydrocephalus with radiological features of disproportionately enlarged subarachnoid space hydrocephalus (DESH). A 78-year-old woman with one-year history of gait disturbances, cognitive impairment, and urinary incontinence. Observe the normal CSF flow across the aqueduct on the 3DT2WTSE (SPACE) midsagittal image (a), the enlarged Sylvian fissures associated with narrowing of the sulci over the high convexity, and low callosal angle on the coronal T2-FLAIR image (b). Ventriculomegaly associated with extensive white matter and some focal sulcal dilation changes is seen on $\mathrm{T} 2$ and T2-FLAIR images $(\mathbf{c}, \mathbf{d})$. Reproduced from Rovira A. Communicating Hydrocephalus. Normal Pressure Hydrocephalus. In F. Barkhof et al. (eds.), Clinical Neuroradiology, (C) Springer Nature Switzerland AG 2019
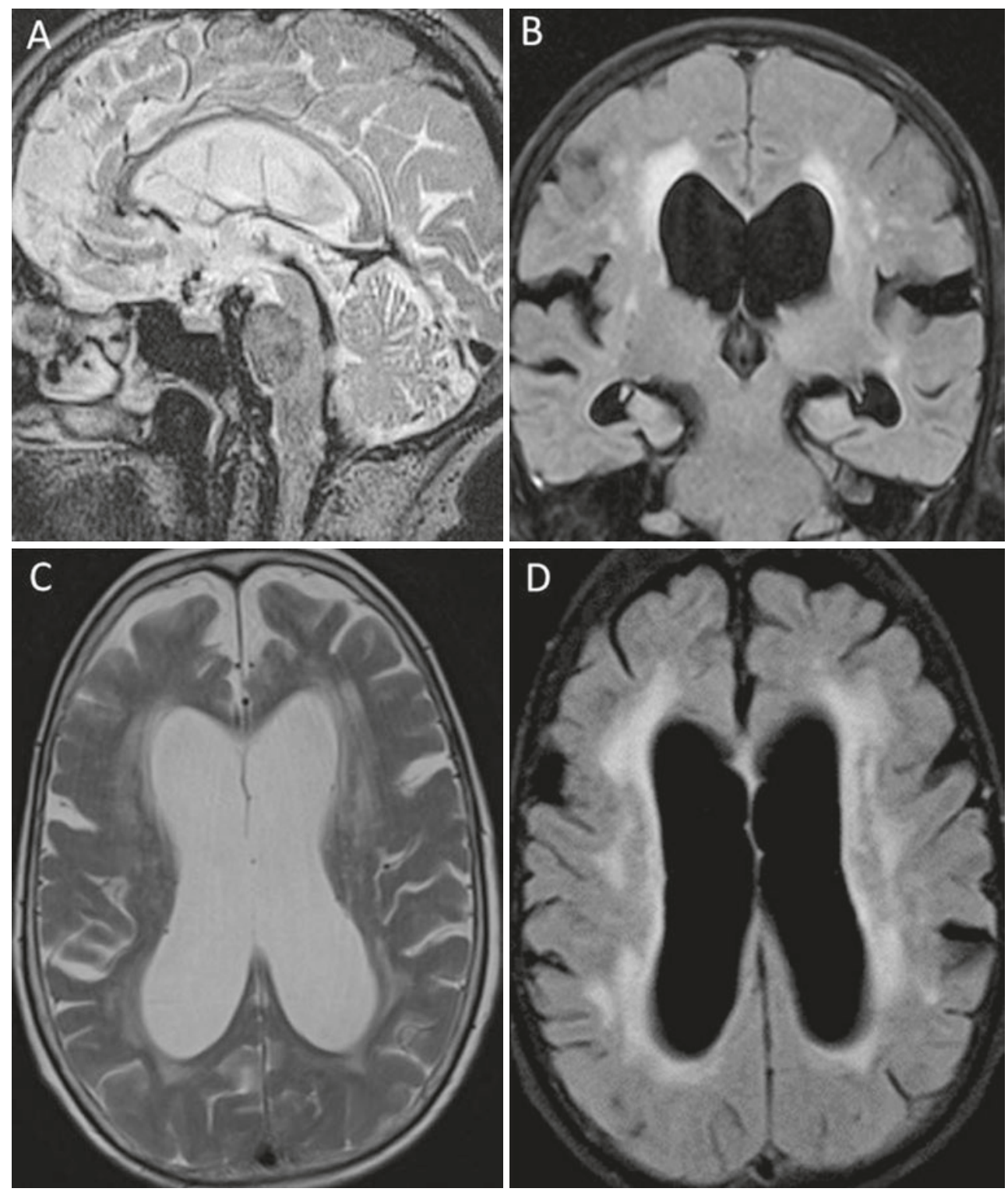
Focal sulcal dilation also occurs in iNPH and should not be mistaken for focal cortical atrophy. These enlarged sulci, which have been considered reservoirs of CSF, usually disappear partially or completely after shunting,

White matter abnormalities (leukoaraiosis) on T2-weighted images, involving both the periventricular and subcortical white matter are a common finding in patients with iNPH, and correlated with a higher frequency of vascular risk factors, such as hypertension. These signal changes are probably related to chronic ischemia as a consequence of cerebral hypoperfusion, and can decrease or even disappear after successful shunt surgery, indicating that at least partially correspond to interstitial edema, as a consequence of an impairment of the glymphatic circulation. The extension of leucoaraiosis in patients with iNPH cannot be used to predict shunt responsiveness.

PC-MRI studies, with a slice positioned in the midsagittal or axial planes, sensitive to CSF movement in the craniocaudal direction (in plane or through plane CSF flow) is a simple way to assess the patency of the aqueduct and to assess the intensity of the pulsatile movement across it. However, the identification and quantification of this CSF movement is not completely specific for the diagnosis of iNPH and does not predict shunt response.

Diversion of CSF is the standard of surgical care for iNPH. Ventriculoperitoneal (VP) shunts are the most popular, while the ventriculoatrial (VA) shunts are rarely implanted because of their more frequent long-term complications. Gait impairment is the symptom that is most responsive to shunting, while cognitive impairment may improve if it is not very severe at the time of intervention, while urinary incontinence improves in 36-90\% of patients.

On CT/MRI studies performed few months after shunt surgery, a mild decrease of the ventricular size is usually seen, associated with CA increased, partial resolution of white matter changes and expansion of sulci over the high convexity sulci (resolution of convexity CSF block).

Not every patient with possible or probable iNPH will be a candidate for shunt surgery, and the risk to benefit ratio has to be assessed on individual bases. Diagnostic test used for selecting patients for shunt surgery includes those based on surgical invasive procedures (CSF dynamics and intracranial pressure monitoring), and those based on morphological or functional MRI studies.

\section{Key Points}

- NPH is characterized by the triad of gait disturbance, mental deterioration, and urinary incontinence, which are associated with enlargement of the ventricular system and normal CSF pressure.

- Structural brain changes grouped under the term "disproportionately enlarged subarachnoid space hydro- cephalus" (DESH), defined as the combination of ventriculomegaly with narrow high convexity and medial subarachnoid spaces and enlarged Sylvian fissures is a key feature to establish the diagnosis of NPH

\subsection{Idiopathic intracranial hypertension (IIH)}

Idiopathic intracranial hypertension (IIH) also known as pseudotumor cerebri or benign intracranial hypertension is a syndrome of elevated intracranial pressure for which there is no identifiable underlying cause. It is most commonly associated with headache and papilledema and more commonly affects females. The symptom of tinnitus is also commonly encountered in patients with IIH. The syndrome typically presents in obese females of child bearing age but can be seen in just about any male or female regardless of body habitus or age. Several medications have been implicated as a cause of secondary $\mathrm{IIH}$; these include tetracycline, minocycline, vitamin A, and retinoids.

The most recently revised diagnostic criteria for the diagnosis of IIH employ a combination of clinical and imaging criteria for diagnosis [15]. IIH has traditionally been a diagnosis of exclusion where neuroimaging was used to simply "rule out" an intracranial mass, hydrocephalus, or dural sinus thrombosis as a cause of elevated intracranial pressure. Many important signs of IIH on MRI and MR venography are now recognized that have dramatically improved our ability to identify the disease in patients independent of clinical suspicion for elevated intracranial pressure.

Diagnostic clinical criteria for IIH include papilledema, normal neurologic examination (except cranial nerve abnormality), normal imaging of the brain parenchyma and meninges, normal CSF composition, and elevated CSF-opening pressure ( $>25 \mathrm{~cm} \mathrm{H}_{2} \mathrm{O}$ ). The diagnosis of $\mathrm{IIH}$ can be considered probable even if there is no papilledema or CSF opening is not elevated provided supportive neuroimaging signs are present.

The neuroimaging signs of elevated intracranial pressure (IP) include empty sella, optic globe deformation seen as flattening of its posterior aspect, papillary protrusion (appreciable optic nerve head protrusion [ \pm enhancement] into the globe correlating with papilledema), tonsillar descent, CSF distention of optic nerve sheath, vertical tortuosity of the intraorbital optic nerve, and stenosis of the distal transverse sinuses. These signs are highly variable in their sensitivity and specificity for predicting the presence of elevated intracranial pressure. Several signs with low sensitivity for increased IP such as papillary enhancement (less than 20\%) have been shown to have very high specificity (99\%), while another such as empty sella has a relatively high sensitivity $(80 \%$ ) but low specificity (less than $80 \%$ ) since it is com- 
monly seen in normal subjects [16]. Stenosis of the distal transverse sinuses remains the most valuable sign for the neuroimaging evaluation of IIH since it has consistently and reproducibly shown the highest (greater than 93\%) sensitivity and specificity. With the advent of gadolinium-enhanced MR venography [17] the transverse sinuses were, for the first time, reliably visualized free from flow artifacts commonly encountered on time-of-flight and phase-contrast MR venographic techniques. Gadolinium-MR venography techniques allowed for the visualization and characterization of the narrowing of the distal transverse sinuses seen in IIH [18]. These most commonly appear as collapse of the dural venous sinuses at or proximal to the transverse sigmoid junction. Alternatively, the stenosis in this region can occasionally be seen due to an enlarged intraluminal arachnoid granulation intrinsically narrowing the lumen.

It must be stressed that the imaging signs described above relate to elevated intracranial pressure and are not exclusive to IIH, as can be present with all other conditions causing elevated IP. The diagnosis of IIH is one of exclusion and all other etiologies must be ruled out. A careful drug history and CSF analysis is mandatory in the work-up of these patients. Moreover, these signs will diminish or resolve following treatment of the elevated pressure [19].

Management of IIH is directed primarily at preserving vision and diminishing the headaches associated with the disease. Treatment typically begins with strategies to improve body habitus and medication (most commonly acetazolamide) and progresses from there as required utilizing surgical procedures of optic nerve fenestration, CSF diversion procedures of ventriculoperitoneal or lumbo-peritoneal shunting, and more recently transverse venous sinus stenting [20]. Since the cause of IIH is not known the treatments can only be directed at the phenotypic or characteristic expression of the disease, i.e., drugs are given which may decrease CSF production or continually remove CSF through extracranial pathways and thus lower IP.

The narrowed transverse sinuses seen in IIH are thought to be a combination of secondary effects arising from elevated IP as well as subsequent superimposition of a positive feedback loop further worsening the IP with venous hypertension. In this way the stenoses are thought to exacerbate the increased IP. Stenting of the stenosed segments breaks the cycle and normalizes venous pressures providing symptomatic relief and protects patients from visual loss. Unfortunately, to date, no randomized control trials exist for evaluation of the efficacy of the surgical options and, therefore, their true efficacy is unknown [21]. Each of the surgical and endovascular options has been shown to have success in treating headache, papilledema, visual loss, diplopia, and tinnitus. Each of these options also carries the risk of complication or need for revision surgery.

Regardless of the cause, the neuroimaging signs of elevated IP (which may relate to IIH) should not be overlooked or overdiagnosed. A diagnosis of IIH cannot be made on imaging grounds alone, and clinical correlation is mandatory to rule out other causes of elevated IP masquerading as IIH.

\section{Key Points}

- IIH has traditionally been a diagnosis of exclusion where neuroimaging is used to simply "rule out" an intracranial mass, hydrocephalus, or dural sinus thrombosis as a cause of elevated IP.

- Stenosis of the distal transverse sinuses remains the most valuable sign for the neuroimaging evaluation of IIH since it has consistently and reproducibly shown the highest sensitivity and specificity for disease.

\subsection{Spontaneous Intracranial Hypotension (SIH)}

Spontaneous intracranial hypotension (SIH) is commonly caused by leakage of CSF from the thecal sac at some point along the spinal canal. The leakage of CSF causes variable symptoms, the most characteristic of which is a positional headache and is commonly associated with typical intracranial MRI features, such as venous distension, pachymeningeal thickening and enhancement, pituitary enlargement, effacement of the suprasellar cistern, subdural fluid collection hygroma or hemorrhage, and sagging deformity of the brainstem with or without tonsillar herniation [22-25] (Fig. 2.7).

In patients being considered for a possible diagnosis of SIH imaging of the entire spine with high-resolution T2-weighted sagittal sequences is mandatory. While many SIH patients present with spinal longitudinal extradural collections (SLECs) some do not [26]. These SLECs have a typical appearance and are seen exclusively in SIH patients with dural mechanical tears along the thecal sac. Conversely, patients with more laterally placed CSF leakage beyond the epidural compartment either from a CSF-venous fistula (CVF) or from a distal root sleeve tear do not display SLECs. The presence or absence of a SLEC on MRI is used to proscribe for the prone versus lateral decubitus positioning of the subsequent dynamic myelography [26, 27]. Digital subtraction myelography (DSM) and ultrafast CT myelography [28] are techniques of dynamic myelography which allow for imaging of gravity dependent contrast media as it descends within the thecal sac from site of injection within the lumbar spine into the cervical spinal canal. By obtaining images in this manner the site at which intrathecal contrast first escapes from the thecal sac and enters the epidural space can be identified with certainty.

Imaging and management of patients with suspected SIH has been a difficult arena for diagnostic neuroradiology. Until 
Fig. 2.7 Spontaneous intracranial hypotension. A 35-year-old woman with a 3-month history of positional headache. Observe the typical intracranial MRI features of this condition: sagging deformity of the brainstem on the sagittal T1-weighted image (a), pachymeningeal thickening and enhancement on axial T2 and contrastenhanced T1-weighted images (b, c), and pituitary enlargement on coronal contrast-enhanced T1-weighted images (d)
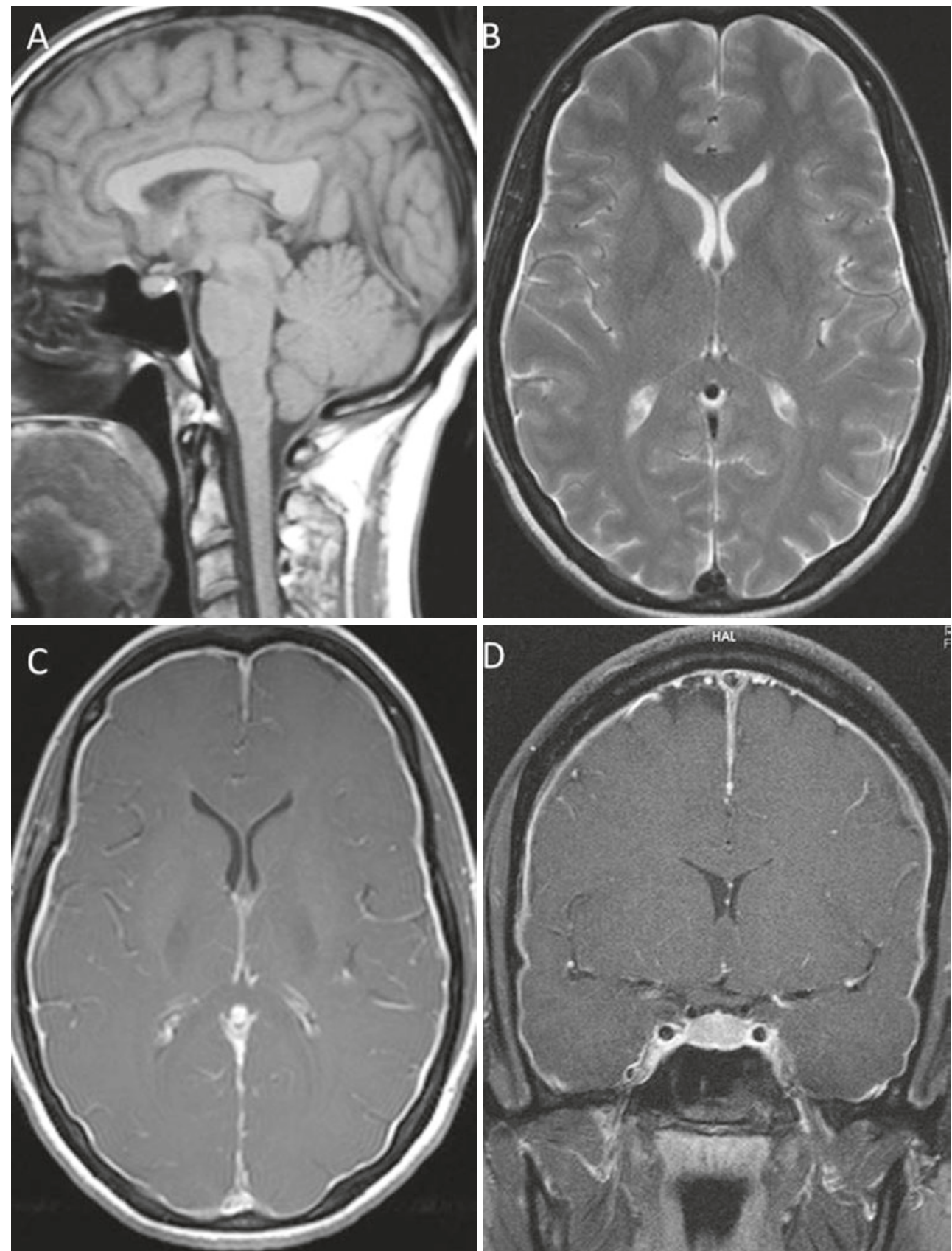

recently there has been a paucity of reliable knowledge disseminated about this syndrome. SIH has only recently been recognized as a syndrome that requires an organized approach by knowledgeable experienced neuroradiologists [26, 29, 30].

In the past, this syndrome has be poorly understood and was thought to be a result of several potential causes including compliance and laxity problems of the thecal sac, low CSF production, and possibly accelerated physiologic loss of CSF. It now appears these leaks are predominantly due to mechanical tears, leaking root sleeves, and CVF. For practicality and ease of description CSF leaks have been classified into four types based on distance from midline [26]. Type 1 is caused by degenerative disc disease creating a mechanical tear in the ventral dura. Patients with these ventral dural punctures are now well recognized as comprising a large population of patients with SIH. All of these patients are SLEC-positive on MRI with or without intracranial findings and show a typical ventral hole in the thecal sac at DSM or dynamic CT. The site of CSF leakage is occasionally seen associated with a calcific "microspur" at the site of a degenerated disk. Unless imaged in a dynamic fashion where the initial flow of contrast is imaged as it travels down the spine the site of leakage (which is commonly microscopic) will be obscured by mixing of contrast in the intradural and extradural compartments above 
and below the hole. A type 2 CSF leak is a less common cause of SIH and is due to a posterolateral dural dehiscent lesion or tear. Type 2 leaks are also associated with SLECs and are not associated with degenerative disk disease. These SLECs may tend to be more posteriorly positioned and their site of origin is commonly incorrectly visualized as a diverticulum adjacent to the root sleeve. In our experience these are more commonly seen in females and appear to be more likely successfully treated with multiple epidural blood patches (EBPs) and bedrest. These are thought to arise from the base of the nerve root (within the axilla) possibly relating to a predisposed thinned or dehiscent area of the dura [29].

If the tear occurs further along the root sleeve adjacent to an arachnoid granulation, where the root sleeve is in close proximity to a venous plexus, then the extravasated fluid is more likely to find its way into a venous channel. In this manner an embryonic pseudomeningocele drains into the venous system and heals aberrantly creating a CVF-a type 3 leak. The type 4 leak is a rare root sleeve leak that does not fistulize into the venous system but rather tracks into and dissipates into the adjacent facial planes. Type 3 and type 4 occur more laterally along the root sleeve beyond the epidural compartment and therefore (1) do not result in a SLEC on spinal MRI and (2) do not respond to nondirected EBP. Focal fibrin patching may have some slowing effect on these leaks, although in our experience has not consistently provided durable cure.

MRI of the brain and spine in patients with SIH allows us to dichotomize patients into those which may benefit from initial EBP prior to dynamic myelography, i.e., SLEC-positive patients (predicting a type 1 or type 2 leak) from those patients in whom nondirected EBP is likely not beneficial, i.e., patients with SLEC-negative imaging (who harbor a type 3 or type 4 leak). SLEC-positive patients (who fail initial EBP) should then undergo DSM in the prone position. This will find over $80 \%$ of the offending type 1 or type 2 tears. If this initial prone DSM is negative, then left and right decubitus DSM should be performed until a leak is found. Conversely SLEC-negative patients should undergo DSM in lateral decubitus position (left then right) to find the offending type 3 or type 4 leaks. Using this type of imaging paradigm an overall success rate of definitively locating the site of CSF leak can be expected in over $90 \%$ of cases.

Dynamic CT myelography (DCTM) is an alternative method to DSM for dynamic myelography [25]. Several authors have reported success in identifying these types of CSF leaks particularly the type 1 and type 2 [26] leaks. It has also been reported on the success of DCTM for type 3 leaks by positioning these patients in the decubitus position to search for CVF. Regardless of the modality chosen it is the spinal MRI which defines these patients as SLEC-positive or SLEC-negative, and proscribes the most efficacious positioning for the subsequent dynamic myelogram. Once found, management can then be directed at the site of CSF leakage. Variable success has been reported with targeted epidural fibrin injections for all types of leaks. The most definitive curative results for treatment of CSF leaks are obtained with surgical repair of the dural tears (types 1 and 2 and 4) and with surgical clipping of the CVF (type 3).

\section{Key Points}

- SIH is commonly caused by leakage of CSF from the thecal sac at some point along the spinal canal.

- In patients being considered for a possible diagnosis of SIH imaging of the entire spine with high-resolution T2-weighted sagittal sequences is mandatory.

- Presence or absence of SLECs on spine MRI is highly useful for guiding dynamic myelographic studies, which are highly sensitive for detecting dural tears.

\subsection{Concluding Remarks}

Structural and functional MRI plays an essential role for understanding the anatomy of the CSF spaces and ventricular system, as well as the hydrodynamics of CSF flow. These principles are important to the comprehension of pathological processes affecting the ventricles and CSF spaces, mainly the different types of hydrocephalus, and disorders associated with abnormal intracranial pressure, such as IIH and SIH. Combination of 3DT2WSS sequences that offer superb anatomical information, and 3DT2WTSE sequences that are highly sensitive to CSF flow, provides an easy evaluation of CSF pathway and circulation to establish the patency of CSF flow and therefore in distinguishing non-communicating from communicating hydrocephalus, which is the first radiological objective in patients with ventriculomegaly.

Diagnosis of IIH and SIH cannot be made exclusively on imaging features, and clinical correlation is mandatory to rule out other causes of elevated or decreased intracranial CSF pressure. In patients being considered for a possible diagnosis of $\mathrm{SIH}$, in addition to brain MRI, imaging of the entire spine with high-resolution T2-weighted sagittal sequences is mandatory, as it provides imaging features that not only support the diagnosis but also guide the subsequent dynamic myelography.

\section{Take Home Messages}

- MRI is the imaging modality of choice in the diagnosis and management of hydrocephalus and other CSF-related diseases.

- Conventional MRI sequences associated with 3DT2WTSE sequences seem to be most efficient MRI strategy for evaluation of hydrocephalus. 
- In complex cases, PC-MRI and 3DT2WSS sequences may add relevant diagnostic information.

- Many signs of elevated intracranial pressure on MRI may suggest the diagnosis of IIH. However, this diagnosis should only be made after careful clinical correlation and exclusion of other etiologies.

- When evaluating patients for a possible diagnosis of SIH, a systematic approach, with proper application of MRI of the brain and spine allows for subsequent efficient work-up and management.

\section{References}

1. Chikly B, Quaghebeur J. Reassessing cerebrospinal fluid (CSF) hydrodynamics: a literature review presenting a novel hypothesis for CSF physiology. J Bodyw Mov Ther. 2013;17:344-54.

2. Chen L, Elias G, Yostos MP, Stimec B, Fasel J, Murphy K. Pathways of cerebrospinal fluid outflow: a deeper understanding of resorption. Neuroradiology. 2015;57:139-47.

3. Kelly EJ, Yamada S. Cerebrospinal fluid flow studies and recent advancements. Semin Ultrasound CT MR. 2016;37:92-9.

4. Agarwal A, Bathla G, Kanekar S. Imaging of communicating hydrocephalus. Semin Ultrasound CT MR. 2016;37:100-8.

5. Kartal MG, Algin O. Evaluation of hydrocephalus and other cerebrospinal fluid disorders with MRI: an update. Insights Imaging. 2014;5:531-41.

6. Maller VV, Gray RI. Noncommunicating hydrocephalus. Semin Ultrasound CT MR. 2016;37:109-19.

7. Barami K, Chakrabarti I, Silverthorn J, et al. Diagnosis, classification and management of fourth ventriculomegaly in adults: report of 9 cases and literature review. World Neurosurg. 2018;116:e709-22.

8. Kahle KT, Kulkarni AV, Limbrick DD, Warf BC. Hydrocephalus in children. Lancet. 2016;387(10020):788-99.

9. Krishnan P, Raybaud C, Palasamudram S, Shroff M. Neuroimaging in pediatric hydrocephalus. Indian J Pediatr. 2019;86:952-60.

10. Dincer A, Ozek MM. Radiologic evaluation of pediatric hydrocephalus. Childs Nerv Syst. 2011;27:1543-62.

11. Bradley WG Jr. CSF flow in the brain in the context of Normal pressure hydrocephalus. AJNR Am J Neuroradiol. 2015;36:831-8.

12. Bradley WG Jr. Magnetic resonance imaging of normal pressure hydrocephalus. Semin Ultrasound CT MR. 2016;37:120-8.

13. Virhammar J, Laurell K, Cesarini KG, Larsson EM. Preoperative prognostic value of MRI findings in 108 patients with idiopathic normal pressure hydrocephalus. AJNR Am J Neuroradiol. 2014;35:2311-8.

14. Mori E, Ishikawa M, Kato T, et al. Japanese Society of Normal Pressure Hydrocephalus. Guidelines for management of idiopathic normal pressure hydrocephalus: second edition. Neurol Med Chir (Tokyo). 2012;52:775-809.

15. Friedman DI. Contemporary management of the pseudotumor cerebri syndrome. Expert Rev Neurother. 2019;19:881-93.

16. Agid R, Farb RI, Willinsky RA, Mikulis DJ, Tomlinson G. Idiopathic intracranial hypertension: the validity of cross-sectional neuroimaging signs. Neuroradiology. 2006;48:521-7.

17. Farb RI, Scott JN, Willinsky RA, Montanera WJ, Wright GA, ter Brugge KG. Intracranial venous system: gadolinium-enhanced three-dimensional MR venography with auto-triggered elliptic centric-ordered sequence-initial experience. Radiology. 2003;226:203-9.

18. Farb RI, Vanek I, Scott JN, et al. Idiopathic intracranial hypertension: the prevalence and morphology of sinovenous stenosis. Neurology. 2003;60:1418-24.

19. Bidot S, Saindane AM, Peragallo JH, Bruce BB, Newman NJ, Biousse V. Brain imaging in idiopathic intracranial hypertension. J Neuroophthalmol. 2015;35:400-11.

20. Friedman DI, Liu GT, Digre KB. Revised diagnostic criteria for the pseudotumor cerebri syndrome in adults and children. Neurology. 2013;81:1159-65.

21. Kalyvas AV, Hughes M, Koutsarnakis C, et al. Efficacy, complications and cost of surgical interventions for idiopathic intracranial hypertension: a systematic review of the literature. Acta Neurochir. 2017;159:33-49.

22. Schievink WI, Maya MM, Jean-Pierre S, Nuno M, Prasad RS, Moser FG. A classification system of spontaneous spinal CSF leaks. Neurology. 2016;87:673-9.

23. Holbrook J, Saindane AM. Imaging of intracranial pressure disorders. Neurosurgery. 2017;80:341-54.

24. Kranz PG, Amrhein TJ, Choudhury KR, Tanpitukpongse TP, Gray L. Time-dependent changes in Dural enhancement associated with spontaneous intracranial hypotension. AJR Am J Roentgenol. 2016;207:1283-7.

25. Mokri B. Spontaneous intracranial hypotension. Continuum (Minneap Minn). 2015;21(4 Headache):1086-108.

26. Farb RI, Nicholson PJ, Peng PW, et al. Spontaneous intracranial hypotension: a systematic imaging approach for CSF leak localization and management based on MRI and digital subtraction Myelography. AJNR Am J Neuroradiol. 2019;40:745-53.

27. Schievink WI, Maya MM, Moser FG, et al. Lateral decubitus digital subtraction myelography to identify spinal CSF-venous fistulas in spontaneous intracranial hypotension. J Neurosurg Spine. 2019;31(6): 1-4.

28. Thielen KR, Sillery JC, Morris JM, et al. Ultrafast dynamic computed tomography myelography for the precise identification of high-flow cerebrospinal fluid leaks caused by spiculated spinal osteophytes. J Neurosurg Spine. 2015;22:324-31.

29. Dobrocky T, Mosimann PJ, Zibold F, et al. Cryptogenic cerebrospinal fluid leaks in spontaneous intracranial hypotension: role of dynamic CT Myelography. Radiology. 2018;289:766-72.

30. Dillon WP. Challenges in the diagnosis and treatment of spontaneous intracranial hypotension. Radiology. 2018;289:773-4.

Open Access This chapter is licensed under the terms of the Creative Commons Attribution 4.0 International License (http://creativecommons. org/licenses/by/4.0/), which permits use, sharing, adaptation, distribution and reproduction in any medium or format, as long as you give appropriate credit to the original author(s) and the source, provide a link to the Creative Commons license and indicate if changes were made.

The images or other third party material in this chapter are included in the chapter's Creative Commons license, unless indicated otherwise in a credit line to the material. If material is not included in the chapter's Creative Commons license and your intended use is not permitted by statutory regulation or exceeds the permitted use, you will need to obtain permission directly from the copyright holder.

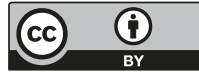

